\title{
Osteocalcin Induces Proliferation, Promotes Differentiation and Decreased IL-6 production Through Activation of GPRC6A in C2C12 Myoblast Cells
}

\author{
Suifeng Liu ${ }^{1}$, Lei Wen ${ }^{2}$, Zaijin Jian ${ }^{3}$, Feng Gao ${ }^{1}$ \\ ${ }^{1}$ Department of cardiology, Zhongshan Hospital, Xiamen University, China, ${ }^{2}$ Department of Traditional Chinese \\ Medicine, Medical college, Xiamen University, Xiamen, China, ${ }^{3}$ Department of Geriatrics, The Second Xiangya \\ Hospital, Central South University, Changsha, China
}

Background: Sarcopenia is an important geriatric syndrome. Despite the clinical significance of sarcopenia, the molecular pathways underlying it remain elusive. Here we explored the promotive effects of ucOC in proliferation and differentiation processes in $\mathrm{C} 2 \mathrm{C} 12$ myoblasts, decreased IL-6 production in $\mathrm{C} 2 \mathrm{C} 12$ myotubes as well as the possible signalling pathways involved. Methods: The effects of exogenous ucOC on $\mathrm{C} 2 \mathrm{C} 12$ myoblasts proliferation were assessed using CCK8 and immunohistological staining assays. The levels of Akt, phosphorylated- Akt (p-Akt), P38, and phosphorylated-P38 (p-P38) were measured by Western Blotting. The effects of ucOC on myoblast differentiation were quantified by morphological analysis. A silencing experiment was conducted in which the expression of GPRC6A in C2C12 myoblasts was modified. The expression of GPRC6A, myosin heavy chain (MyHC) and the related ERK1/2 signalling pathway in $\mathrm{C} 2 \mathrm{C} 12$ myoblasts were monitored by qRT-PCR and Western Blotting. IL-6 production in C2C12 myotubes were detected using the method of ELISA. Results: We showed that treatment with exogenous ucOC stimulated the priming of $\mathrm{C} 2 \mathrm{C} 12$ myoblasts proliferation. Inhibition of Akt phosphorylation by wortmannin or inhibition of P38 MAPK phosphorylation by SB203580 decreased C2C12 cell proliferation. Wortmannin also reduced P38 MAPK phosphorylation, whereas SB203580 did not affect Akt activation. Besides, ucOC promoted C2C12 myoblast differentiation. Inhibition of ERK1/2 phosphorylation with U0126 decreased C2C12 cell differentiation. Furthermore, ucOC played a role of anti-inflammation and decreased IL-6 production in isolated C2C12 myotubes. Finally, GPRC6A expression was substantially increased after ucOC treatment of C2C12 cells. GPRC6A silencing inhibited Akt, P38 MAPK phosphorylation in $\mathrm{C} 2 \mathrm{C} 12$ cells, and ERK1/2 phosphorylation in C2C12 myotubes; GPRC6A silencing also decreased cell proliferation, decreased cell differentiation, and increased IL-6 expression. Conclusions: The present data suggest that ucOC induces myoblast proliferation, enhances myogenic differentiation and decreased IL-6 production via a mechanism involving GPRC6A. We believe that our data support the idea that ucOC plays a major role in sarcopenia by promoting myoblast cellular function and anti-inflammatory property.

Supported by the by grants from the National Natural Science Foundation of China (grant No. 81272203) and the Natural Science Foundation of Fujian Province of China (grant No. 2016J01624). 